\title{
Statin-induced cytotoxicity through a mevalonate-independent mechanism
}

\author{
Jae Won Choi ${ }^{1}$, Hyoshik Shin ${ }^{1}$, Jichang Seong ${ }^{1}$, Junghan Yoon ${ }^{2}$
${ }^{1}$ Pharmacology, Yonsei University Wonju College of Medicine, Korea, ${ }^{2}$ Internal Medicine, Yonsei University Wonju College of Medicine, Korea

Statins are widely-used anti-hyperlipidemics that inhibit 3-hydroxy-3-methyl glutaryl-coenzyme A (HMG-CoA) reductase to decrease intracellular levels of mevalonate. It has been suggested that the side effects caused by statins are mostly induced by deprivation of intracellular metabolic intermediates derived from mevalonate. However, the pathophysiological mechanisms underlying myalgia, a common side effect with an incidence rate of $10-30 \%$, and other rare, but serious, side effects such as rhabdomyolysis, new-onset diabetes, acute renal injury, and peripheral neuropathy, are not yet fully understood. We hypothesized that statins can disrupt cellular functions directly by interacting with target molecules other than HMG-CoA reductase. To verify this hypothesis, we observed the direct effects of statins at a high concentration (1 $\mathrm{mM}$ ) on cells. In C6 glioma cells, either lovastatin or simvastatin increased intracellular ROS levels significantly after 30 minutes of treatment, whereas pravastatin did not. The increase in ROS production was more prominent when the cells were treated with an inactive lactone form of statins. At two hours after treatment, the ROS production returned to baseline values. The sudden increase in ROS production could be prevented by organic anions, such as lactic acid, which indicated that intracellular statins, which were transported into cells by MCT and/or OATP, triggered ROS production. The increase in ROS levels could be suppressed by antioxidants, but not by mevalonate, diphenyleneiodonium, or Nnitro-L-arginine methyl ester. The LDH assay showed that approximately 51 and $86 \%$ of cells were dead at 12 hours after lovastatin and simvastatin treatment, respectively; however, pravastatin did not induce cell death. The antioxidants partially prevented statin-induced cell death, but mevalonate could not. After lovastatin treatment, mitochondrial membrane potential (MMP) disruption could be observed within one hour. Subsequently, MMP returned to the normal levels, but was disrupted again after five hours of treatment. The initial phase of MMP disruption was suppressed by cyclosporine A (CsA) or 4,4'-diisothiocyanostilbene-2,2'-disulfonic acid (DIDS). The late phase of MMP disruption was suppressed by DIDS for up to 16 hours, but was augmented by CsA. Our data show that statins can exert direct cytotoxicity by interacting with target molecules other than HMG-CoA reductase. 